OPEN ACCESS

Edited by:

Ondrej Viklicky,

Institute for Clinical and Experimental

Medicine (IKEM), Czechia

Reviewed by:

Hongwei Chen,

University of Michigan,

United States

Petra Hruba,

Institute for Clinical and Experimental

Medicine (IKEM), Czechia

*Correspondence:

Ulrich Martin

martin.ulrich@mh-hannover.de

Specialty section:

This article was submitted

to Translational Medicine,

a section of the journal

Frontiers in Medicine

Received: 14 September 2017 Accepted: 30 November 2017

Published: 14 December 2017

Citation:

Martin U (2017) Therapeutic Application of Pluripotent Stem

Cells: Challenges and Risks.

Front. Med. 4:229.

doi: 10.3389/fmed.2017.00229

\section{Therapeutic Application of Pluripotent Stem Cells: Challenges and Risks}

\author{
Ulrich Martin* \\ Leibniz Research Laboratories for Biotechnology and Artificial Organs (LEBAO), Department of Cardiothoracic, \\ Transplantation and Vascular Surgery, REBIRTH Cluster of Excellence, German Center for Lung Research, \\ Hannover Medical School, Hannover, Germany
}

Stem-cell-based therapies are considered to be promising and innovative but complex approaches. Induced pluripotent stem cells (iPSCs) combine the advantages of adult stem cells with the hitherto unique characteristics of embryonic stem cells (ESCs). Major progress has already been achieved with regard to reprogramming technology, but also regarding targeted genome editing and scalable expansion and differentiation of iPSCs and ESCs, in some cases yielding highly enriched preparations of well-defined cell lineages at clinically required dimensions. It is noteworthy, however, that for many applications critical requirements such as the targeted specification into distinct cellular subpopulations and a proper cell maturation remain to be achieved. Moreover, current hurdles such as low survival rates and insufficient functional integration of cellular transplants remain to be overcome. Nevertheless, PSC technologies obviously have come of age and matured to a stage where various clinical applications of PSC-based cellular therapies have been initiated and are conducted.

Keywords: induced pluripotent stem cells, embryonic stem cells, cell product, cellular therapy, risks, good medical practice, clinical translation

\section{INTRODUCTION}

Modern medical technologies with the countless number of available drugs, new diagnostic procedures, and therapeutic applications including complex surgical interventions greatly contributed to a substantially increased life expectancy not only in industrialized countries. On the other hand, the increasing frequency of chronic and age-related diseases, lifestyle diseases, or diseases caused by environmental pollution have created new challenges for medical research, including a clear need to develop novel regenerative therapies.

Stem-cell-based concepts are widely considered as promising, but recent experimental and clinical studies applying various adult stem cells, for instance, for cardiac repair (1), have yielded for the most part disappointing results, in many cases without evidence of any considerable functional improvement or therapeutic effects. Major limitations of the various adult stem and progenitor cell types that had been applied include the restricted potential for culture expansion and especially differentiation into the desired therapeutic cell lineages. Whereas the application of adult stem and

Abbreviations: (h)iPSCs, (human) induced pluripotent stem cells; hPSCs, (human) pluripotent stem cells; ESCs, embryonic stem cells; RPE, retinal pigment epithelium; HLA, human leukocyte antigen. 
progenitors is promising or has already proven successful in case of tissue types that show considerable endogenous potential for regeneration, such as bone or the hematopoietic system (2), treatment of diseases that affect less regenerative organs, such as the brain and the heart (1), has not shown significant therapeutic effects, or the observed therapeutic benefit was due to paracrine effects. Another limitation for experimental evaluation and clinical application of different adult stem and progenitor cells is the typical inability to achieve efficient targeted genome engineering, for instance, to introduce therapeutic transgenes or to correct disease-causing mutations, followed by subsequent isolation of defined transgenic single cell clones. Instead, introduction of therapeutic or reporter transgenes into adult stem and progenitor cells is usually achieved through viral vectors with their wellknown drawbacks, including limited transient transgene expression, insertional mutagenesis, and in vivo immunogenicity (3).

The availability of human pluripotent stem cells (PSCs), including embryonic stem cells (ESCs) and induced pluripotent stem cells (iPSCs) (4), with their far-reaching potential for proliferation and differentiation, now offers novel opportunities for basic research, disease modeling and drug discovery (5), as well as for the development of cellular therapies.

Intense research has driven forward dramatic progress in virtually all areas of iPSC technology. This includes highly efficient technologies for the transgene-free reprogramming of easily accessible cell sources, such as blood (6), efficient and safe sitespecific genetic engineering of iPSCs (7), and the development of technologies for the expansion and differentiation of iPSCs at clinical scale (8). Importantly, also intense research on safety issues of iPSC-based cell transplants, the most critical aspect for clinical applications, is ongoing (9).

The Nobel Prize in 2012 was awarded to S. Yamanaka in particular because the use of iPSCs in disease modeling was already a reality by this time and their immense usefulness in drug development already became obvious. By contrast, and although one single trial using ESCs for treatment of spinal cord injury had already been initiated (10), broad clinical application of PSC-based cellular therapies were not foreseeable in 2012. Five years later, this has changed. A considerable number of ESCbased trials are currently conducted or are recruiting patients. In Japan, people suffering from macular degeneration became the first patients to be treated with iPSC-derived retinal pigment epithelium (RPE), using both autologous and human leukocyte antigen (HLA)-matched allogeneic cells (11). Worldwide, a series of clinical studies are in preparation or ongoing for applications of therapeutic derivatives of PSCs for treatment of macular degeneration, diabetes, neurological disorders, and heart failure (Table 1).

\section{GENERAL TECHNOLOGICAL REQUIREMENTS FOR PSC-BASED CELLULAR THERAPIES}

Meanwhile, many hurdles and limitations for production of clinically applicable ESC and iPSC derivatives have already been overcome. Transgene-free iPSCs can be efficiently derived from easily accessible cell sources such as blood $(14,15)$. ESCs and iPSCs can now be expanded in scalable suspension culture (8) and large numbers of human PSCs can be produced in fully controlled bioreactors in defined culture media $(16,17)$. Moreover, sequential inhibition and activation of molecular differentiation pathways has, for the first time, allowed a targeted, more robust and efficient differentiation of human ESCs and iPSCs (18-20). In the meantime, small molecules instead of recombinant proteins are used in such protocols, which have greatly improved the efficiency of differentiation into specific lineages (21-24). The introduction of small molecules as an activator or inhibitor of molecular key pathways has facilitated the development of scalable protocols that are relatively inexpensive and more robust. For various cell lineages, a bottle neck is still the maturation of the PSC-derived cells of interest. It is always a matter of debate whether it is better to transplant mature cells with full functionality (e.g., hepatocytes) or less mature ones that may still have some plasticity and may lead to better structural and functional integration of the graft (e.g., cardiomyocytes with embryonic or fetal phenotype). In other cases, in particular in organs and tissue with substantial stem cell-based regenerative potential (e.g., lung epithelium), it may even be necessary to apply tissue-specific stem cells derived from PSCs in order to achieve long-term functionality.

Also important, highly efficient protocols for a site-specific (and, thus, relatively safe) introduction of transgenes or correction of disease-specific mutations by homologous recombination have been developed based on engineered nucleases, including Zinc finger- and TALE (transcription activator-like effector)-nucleases as well as the CRISPR/Cas9 (clustered regularly interspaced short palindromic repeats; CRISPR-associated proteins) system (25). Utilizing these tools, it has been possible to develop protocols for footprintless gene editing without the need for antibiotic selection (26). Such techniques are of key importance for preclinical animal studies and future cellular therapies as they allow, for example, the correction of disease-related mutations in autologous cells (7) or the well-defined expression of therapeutic transgenes or reporter genes in safe harbor sites that facilitate monitoring of graft survival, functional integration, and distribution in small and large animals (27). Other applications include the targeted introduction of selection genes to enable enrichment of pure cell lineages prior to transplantation (28) or the killing of cellular grafts in case of tumor formation through inducible expression of suicide genes (29).

Another critical hurdle for future stem cell-based therapies that is still not sufficiently addressed is the mode of cell delivery. For most cellular therapeutics, optimal cell application routes and approaches have not been developed yet. In case of heart repair after myocardial infarction, which is a good example for the technical hurdles that have to be overcome, intracoronary infusion was favored for application of bone marrow cells. In this case, it can be observed that a considerable number of the relatively small bone marrow cells is able to cross the vascular wall and is subsequently detectable in the heart muscle (30), but does not form functional myocardium. In the case of the much larger PSC-derived cardiomyocytes, these cells are injected directly into the myocardium, either surgically or via a catheter $(31,32)$. One shortcoming of this approach is the extensive loss 
TABLE 1 | Therapeutic application of embryonic stem (ES) cell and induced pluripotent stem (iPS) cell products: clinical trials.

\begin{tabular}{ll}
\hline Study title & $\begin{array}{l}\text { Registration Status } \\
\text { number }\end{array}$
\end{tabular}

number

(Estimated) (Estimated) Country Comments

enrollment study

completion

date

\section{ES CELLS}

Cardiac diseases

Transplantation of human embryonic stem cell (ESC)-derived progenitors in severe heart

failure (ESCORT)

\section{Ophthalmic diseases}

Stem cell therapy for outer retinal degenerations

Phase I study; first patients treated

(12), recruiting further patients

$\begin{array}{ll}\text { NCT02903576 } & \text { Phase I/II study, recruiting } \\ \text { NCT02749734 } & \text { Phase I study; recruiting }\end{array}$

epitheliums (RPEs) in treatment of macular degeneration diseases

Subretinal transplantation of RPEs in treatment of age-related macular degeneration diseases

Treatment of dry age-related macular degeneration disease with RPE derived from human ESCs

NCT02755428 Phase I study; recruiting

NCT03046407 Early phase I study; recruiting

Observational study; recruiting

$11001491 \quad$ completed

Preliminary clinical studies of stem cells therapy for retinal degeneration diseases

ChiCTR-

Observational study; recruiting

$\mathrm{OCH}-14005060$

The clinical trial of human ESC-derived epithelial cells transplantation in the treatment

of severe ocular surface diseases

Clinical study of subretinal transplantation of human embryo stem cell-derived RPEs in

treatment of macular degeneration diseases

Clinical study of subretinal transplantation of clinical human embryonic stem cells-derived

retinal pigment epitheliums (hESC-RPEs) in treatment of dry age-related macular

degeneration diseases

Safety and efficacy study of OpRegen for treatment of advanced dry-form age-related

macular degeneration

Safety and tolerability of MA09-hRPE cells in patients with Stargardt's macular

dystrophy (SMD)

A Phase I/lla, open-label, single-center, prospective study to determine the safety and tolerability of sub-retinal transplantation of human ESC-derived retinal pigmented epithelial

(MA09-hRPE) cells in patients with advanced dry age-related macular degeneration (AMD)

A study of implantation of RPE in subjects with acute wet age-related macular degeneration

A follow up study to determine the safety and tolerability of sub-retinal transplantation of

hESC-RPE cells in patients with SMD

ChiCTR

Observational study; recruiting

OCB-15005968

ChicTR-

OCB-15006423

ChiCTR-

OCB-15007054

Observational study

Observational study; recruiting

10

10

NCT02286089 Phase I/II study, recruiting

NCT01625559

Phase I study; not recruiting (exact status unknown)

Phase $1 / /$

NCT01691261 Phase I study; active but not

recruiting

NCT02941991

Safety and tolerability of sub-retinal transplantation of hESC-RPE cells in patients with SMD $\quad$ NCT01469832 $\quad$ Phase I/II study; completed (13)

Safety and tolerability of sub-retinal transplantation of hESC-RPE cells in patients with SMD $\quad$ NCT01469832 Phase I/ll study; completed (13)

to 5 years of a Phase I/ll study;

active, not recruiting

Phase I/II study; completed (13)

Sep 2015

USA/UK 
date

Safety and tolerability of sub-retinal transplantation of hESC-derived RPE (MA09-hRPE) cells NCT01344993 in patients with advanced dry age-related macular degeneration (Dry AMD)

Sub-retinal transplantation of hESC-derived RPE (MA09-hRPE) cells in patients with SMD

Long term follow up of sub-retinal transplantation of hESC-derived RPE cells in SMD patients NCT02445612

Phase I/II study; completed (13)

13 Aug 2015

USA

Long term follow up of sub-retinal transplantation of hESC-derived RPE cells in

Phase I/l study, completed (13)

13 Aug 2015 USA

patients with $A M D$

NCT02463344 Observational study; follow-up

Observational study; long-term

Dec 2019 USA

active, not recruiting

study to NCT01344993; active, not recruiting

Study of subretinal implantation of human ESC-derived RPE cells in advanced dry AMD $\quad$ NCT02590692

Phase I/II study; recruiting

11

Dec 2019

A Safety surveillance study in subjects with macular degenerative disease treated with human NCT03167203 ESC-derived retinal pigment epithelial cell therapy

Phase I/II study; not yet recruiting

Sep 2022

USA

\section{Neurological disorders}

Safety and efficacy study of human ESC-derived neural precursor cells in the treatment of

Parkinson's disease

Dose escalation study of AST-OPC1 in spinal cord injury

NCT03119636 Phase I/ll study; recruiting

Dec 2029

USA

Diabetes

A safety, tolerability, and efficacy study of $\mathrm{VC}-01^{\mathrm{TM}}$ combination product in subjects with type

I diabetes mellitus

Three year follow-up safety study in subjects previously implanted with VC-01 TM

NCT02302157 Phase I/II study; recruiting

50

Dec 2020 China

A safety and tolerability study of $\mathrm{VC}-02^{\mathrm{TM}}$ combination product in subjects with type 1

diabetes mellitus

NCT02239354

Phase I/II study; active, not

recruiting

NCT02939118 Observational study; enrolling

participants by invitation

A safety, tolerability, and efficacy study of $\mathrm{VC}-02^{\mathrm{TM}}$ combination product in subjects with

NCT03162926 Phase I study; recruiting

NCT03163511 Phase I/ll study; recruiting

type 1 diabetes mellitus and hypoglycemia unawareness

\section{Blood diseases}

Thalassemia treatment based on the stem cell technology

NCT032224

Interventional study; enrolling participants by invitation

35 Dec $2018 \quad$ USA

\section{Ophtalmic diseases}

Pilot safety study of iPSC-based intervention for wet-type AMD

Phase I study; one patient don recruitment terminated in 2015

65

Jan 202

USA

$20 \quad$ Nov $2021 \quad$ USA

15 Jun 2018 USA

Canada

55 Dec 2020 USA

USA

Production of iPSC-derived RPE cells for transplantation in AMD

NCT02464956 Observational study; recruiting

status unknown

$2 \quad$ Dec $2017 \quad$ China

The above table is without any claim of completeness.

n.a., not available. 
of injected cells directly after application through the injection channel (33). Moreover, although new well-structured functional myocardium is obviously formed, the already existing scar tissue is not replaced and may limit the graft-based functional improvement or may cause arrhythmias (32). Replacement of the whole infarction scar through a tissue engineered myocardial graft that is produced from PSCs may overcome these limitations. However, the problem of proper vascularization of such large dimensional patches has not been solved yet.

\section{RISKS AND HURDLES ASSOCIATED WITH PSC-BASED CELLULAR THERAPIES}

Besides the development of robust and scalable differentiation protocols, research on safety issues concerning ESC- and iPSCbased cell transplants, including the appearance of genetic abnormalities (34), is probably the most critical aspect for future clinical application. It is necessary to carefully investigate to what extent mutations are already present in the source cells (35), or if they are generated during reprogramming, or are enriched during subsequent hiPSC expansion, which is mandatory for many therapeutic applications. In addition, standard operation procedures (SOPs) are required to routinely check PSCs and their derivatives for abnormalities prior to clinical use. However, many aspects have to be considered during assessment of PSCrelated tumor risks. It may well be that risks associated with genetic aberrations are overestimated. It should be emphasized that the detected large number of genetic variations and aberrations in PSCs is somehow misleading since these cells have been investigated for such abnormalities and polymorphisms in much more detail than any other non-tumor cell type. Remarkably, also somatic tissue contains a multitude of genetic variations which do not cause cancer (36). Moreover, the therapeutic tumor risk caused by an individual mutation in PSCs depends to a high degree on the cell type to be transplanted. For instance, terminally differentiated cardiomyocytes are very unlikely to give rise to tumor cells, even if they contain several cancer-related mutations.

Nevertheless, future preclinical risk assessment will definitely require extensive research on tumor and disease risks associated with therapeutic applications of PSC derivatives. Finally, PSCderived therapeutic cell products will have to undergo extensive characterization, most likely including laborious and relatively expensive techniques such as karyotyping, array comparative genomic hybridization analyses, and whole exome sequencing.

Of course, there are further risks related to the specific graft and its function, the application procedure, the tissue and organ to be treated, and the individual patient. These include, for instance, life-threatening arrhythmia after transplantation of PSC-derived cardiomyocytes, infection risks caused by the application procedure, excessive inflammation due to donor cell death, and immune reactions against transgenic grafts or graft vs. host reactions in case of hematopoietic transplants. Clearly, all these risks have to be considered during development of a regulatory framework for PSC-based cellular therapies.

\section{GENERAL REGULATORY REQUIREMENTS}

For clinical applications of ESC and especially iPSC-derived cellular products, the development of more general and therapy/ product-specific regulatory guidelines is just in its infancy. At this point, risks are widely unknown. Available reprogramming, culture, gene editing, differentiation, and application techniques are continually being developed and improved, and assays for risk assessment and potency assays have to be defined in close interaction between regulatory authorities, researchers, manufacturers and clinicians.

Doubtless to say, the requirements for standardization and safety of cellular PSC products are demanding. Remarkably, in contrast to most adult stem/progenitor cell types, animalcomponent-free defined culture media, and matrix formulations have already been developed and iPSC lines have been produced under such conditions (6). Meanwhile, such media are applied in many laboratories for reprogramming and culture expansion. Also, first good medical practice (GMP)-grade culture media are commercially available and other GMP components (e.g., reprogramming kits) are under development by different manufacturers. Although first GXP-conform protocols have already been developed in individual cases, lots off effort will be required for development of SOPs.

Besides development and determination of defined protocols for cell production, product characterization, and clinical application, risk assessment will be of utmost importance for PSC products in general and for individual cell products. The individual risks of teratoma formation due to contaminated undifferentiated cells have to be assessed and minimized. Also, further research focusing on the genetic stability of PSCs is required, especially as genetic abnormalities in their therapeutic derivatives might harbor the risk of tumor formation. However, it is noteworthy to mention that despite leukemia and other tumor cells, there is no other cell type whose genetic integrity, including the occurrence of chromosomal aberrations and nucleotide polymorphisms, has been explored in so much detail as human PSCs. Based on the existing data and ongoing research in the field, suitable assays for risk assessment and subsequent routine characterization of PSC-based cell products will have to be defined.

\section{THERAPEUTIC APPLICATION OF PSC- BASED CELL PRODUCTS: CURRENT CLINICAL TRIALS}

\section{PSCs for Treatment of Ophthalmic Diseases}

The vast majority of the current clinical trials on transplantation of PSC-based cell products aim at cellular therapy of macular degeneration (Table 1). Because the eye is known as an immuneprivileged site, the majority of trials that are currently planned or in process use established allogeneic ESC lines. Interestingly more than 20 ESC-based trials of ophthalmic diseases have been registered in various countries including Brazil, China, Israel, Korea, UK, and USA. Among them, single trials have already 
been completed, and others are currently active or are recruiting patients.

Following approval by the Japanese authorities, two patients were also enrolled in a clinical trial for transplantation of autologous iPSC-derived retinal cells. This is the first clinical trial that is based on an iPSC-based transplant. The first patient underwent treatment for macular degeneration using a hiPSCderived retinal cell sheet in September 2014 (37). One year after transplantation, there were no serious complications and no unexpected proliferation or sign of local or systemic malignant disease. The transplanted iPSC-RPE sheet showed no sign of rejection until December 2016. Moreover, the best corrected visual acuity of the treated eye did not improve or worsen (http:// www.riken-ibri.jp/AMD/english), and it was concluded that the iPSC-based autologous transplantation was safe and feasible in this patient (38).

The second patient enrolled was not treated because of three copy number variations in one patient's iPSC line that were not detectable in the patient's original fibroblasts, but that would probably impact expression of affected and surrounding genes (38). The fact that this decision was made, although the conducted safety assays did not provide any evidence that the iPSC-derived RPE cells can be tumorigenic, demonstrates that the iPSC field is well aware of the importance of safety issues. Meanwhile, a second patient has been treated with allogenic iPSC-derived HLA-matched cells (11).

\section{PSCs for Treatment of Neurological Disorders}

The world's first industry-sponsored clinical trial on a cellular therapy based on human ESCs was initiated in 2010 by Geron, Menlo Park, California, to explore whether hESCs can help nerves to regrow in cases of spinal-cord injury. In 2011, Geron abruptly stopped its stem-cell-therapy programs, including this trial, to focus on its anti-cancer portfolio (39). Even though the trial has officially ended, participants will be monitored for 15 years. In 2017, it was reported that in five treated patients the cell transplant was well tolerated without any detectable immune response despite HLA mismatches, even 10 months after termination of the transient low-dose immunosuppression. The trial was designed as an efficacy study with its low cell dose (5-10× below predicted efficacious range) and suboptimal patient population. However, there was no evidence of significant changes in neurological function and no evidence for ascending loss of function from cells or due to cell application procedure (J. Lebkowski, ISSCR Workshop on Clinical Translation, June 13, 2017).

In 2014, a new Californian company, Asterias Biotherapeutics, started to resurrect the above trial. In 2015, the first patient was treated in a "Phase $1 / 2$ a Dose-Escalation Clinical Trial of AST-OPC1 for Complete Cervical Spinal Cord Injury." In 2017, fist preliminary 9-month efficacy data were presented but final data is pending. Despite these clinical trials for treatment of spinal cord injury, one clinical trial in China has been registered focusing on the development of an ESC-based therapy of Parkinson's Disease.

\section{PSCs for Treatment of Diabetes}

Four industry-sponsored clinical trials have been registered for treatment of diabetes applying ESC-derived beta cells. Developed and produced by the Californian company Viacyte, pancreatic progenitor cells are used as an islet cell replacement therapy by being transplanted in a device designed to allow direct vascularization of the cells, or by being encapsulated in an immunoprotected device (VC-01 ${ }^{\mathrm{TM}}$ ).

\section{PSCs for Clinical Heart Repair}

As yet, there is only one registered clinical trial utilizing PSCs for heart repair. The aim of the "ESCORT"-trial was to demonstrate feasibility and safety of the transplantation of hESC-derived progenitors in severe heart failure. It is noteworthy that no ESC-derived cardiomyocytes have been transplanted and that, although reported by the same group for similar non-human primate progenitors (40), it has not been independently confirmed that the transplanted SSEA- $1^{\text {pos }}$ progenitors are able to form functional cardiomyocytes. As yet, treatment of only one patient was reported without complications, such as arrhythmias, tumor formation, or immunosuppression-related adverse events within 3 months of follow-up (12).

However, based on recent advances in relevant technologies including scalable and greatly improved cardiac differentiation efficiencies in defined media (41), different enrichment strategies such as fluorescence-activated cell sorting $(42,43)$ or metabolic enrichment (44) as well as specification of cardiomyocyte subtypes of these cells $(45,46)$, it can be presumed that first clinical trials on transplantation of PSC-derived cardiomyocytes will be initiated in the upcoming years.

\section{PERSPECTIVES}

Cellular therapies based on ESCs and iPSCs are considered as innovative but complex therapeutic concepts. Addressing the elaborate requirements, basic scientists with leading expertise in hPSC technology, experts in clinical process scale up and toxicology testing, veterinarians and clinician scientists experienced in preclinical large animal models, imaging, and clinical translation of innovative therapies, and experts in ethical and legal aspects of the life sciences have to team up for the development of therapeutic applications and cellular products. Clinical trials that involve standard operating procedures for the production of cellular grafts, quality control, and the application procedure have to be developed together with the competent national authorities, such as the FDA (U.S. Food and Drug Administration) or the EMA (European Medicines Agency).

In most cases, biotech companies with experience in GMPcompliant cell processing and production should also be involved to enable successful clinical implementation and commercial translation.

To date, however, there is not much practical experience in the development of hPSC-based therapeutics, and basic regulatory requirements for adult stem cell therapeutics will need to be adapted. This uncertainty leads to a high capital requirement for possible development costs reducing the calculated return on investment. Therefore, most endeavors will at a first stage focus on 
non-routine products for individual patients under the umbrella of the so-called "hospital exemption."

At this stage, it is uncertain when broad clinical application of PSC products can become reality, and whether these applications will be based on patient-specific autologous cells. The production of autologous iPSC-derived cellular therapeutics is generally feasible, but is associated with high costs and would require at least several months for iPSC generation, potential gene editing, differentiation, and quality control.

An alternative approach may be the application of HLAmatched allogeneic iPSC lines from public biobanks, in particular from HLA-homozygous donors, which appears feasible especially in the island nation Japan with its restricted HLA repertoire.

Other approaches enabling the use of allogeneic cells include the induction of peripheral immunological tolerance toward the cell transplant, or the development of PSC lines with substantially reduced allogeneic immunogenicity (47), in particular by

\section{REFERENCES}

1. Jiang $\mathrm{M}, \mathrm{He} \mathrm{B}$, Zhang Q, Ge H, Zang $\mathrm{MH}$, Han $\mathrm{ZH}$, et al. Randomized controlled trials on the therapeutic effects of adult progenitor cells for myocardial infarction: meta-analysis. Expert Opin Biol Ther (2010) 10(5):667-80. doi:10.1517/14712591003716437

2. Goldstein G, Toren A, Nagler A. Transplantation and other uses of human umbilical cord blood and stem cells. Curr Pharm Des (2007) 13(13):1363-73. doi:10.2174/138161207780618759

3. Rothe M, Modlich U, Schambach A. Biosafety challenges for use of lentiviral vectors in gene therapy. Curr Gene Ther (2013) 13(6):453-68. doi:10.2174/ 15665232113136660006

4. Takahashi K, Tanabe K, Ohnuki M, Narita M, Ichisaka T, Tomoda K, et al. Induction of pluripotent stem cells from adult human fibroblasts by defined factors. Cell (2007) 131(5):861-72. doi:10.1016/j.cell.2007.11.019

5. Shi $\mathrm{Y}$, Inoue $\mathrm{H}, \mathrm{Wu} J \mathrm{~J}$, Yamanaka S. Induced pluripotent stem cell technology: a decade of progress. Nat Rev Drug Discov (2017) 16(2):115-30. doi:10.1038/ nrd.2016.245

6. Haase A, Göhring G, Martin U. Generation of non-transgenic iPS cells from human cord blood CD34+ cells under animal component-free conditions. Stem Cell Res (2017) 21:71-3. doi:10.1016/j.scr.2017.03.022

7. Merkert S, Bednarski C, Göhring G, Cathomen T, Martin U. Generation of a gene-corrected isogenic control iPSC line from cystic fibrosis-patient specific iPSCs homozygous for p.Phe508del mutation mediated by TALENs and ssODN. Stem Cell Res (2017) 23:95-7. doi:10.1016/j.scr.2017.07.010

8. Zweigerdt R, Olmer R, Singh H, Haverich A, Martin U. Scalable expansion of human pluripotent stem cells in suspension culture. Nat Protoc (2011) 6(5):689-700. doi:10.1038/nprot.2011.318

9. Weissbein U, Benvenisty N, Ben-David U. Quality control: genome maintenance in pluripotent stem cells. J Cell Biol (2014) 204(2):153-63. doi:10.1083/jcb.201310135

10. Alper J. Geron gets green light for human trial of ES cell-derived product. Nat Biotechnol (2009) 27(3):213-4. doi:10.1038/nbt0309-213a

11. Cyranoski D. Japanese man is first to receive 'reprogrammed' stem cells from another person. Nature (2017). doi:10.1038/nature.2017.21730

12. Menasche P, Vanneaux V, Hagege A, Bel A, Cholley B, Cacciapuoti I, et al. Human embryonic stem cell-derived cardiac progenitors for severe heart failure treatment: first clinical case report. Eur Heart J (2015) 36(30):2011-7. doi:10.1093/eurheartj/ehv189

13. Schwartz SD, Regillo CD, Lam BL, Eliott D, Rosenfeld PJ, Gregori NZ, et al. Human embryonic stem cell-derived retinal pigment epithelium in patients with age-related macular degeneration and Stargardt's macular dystrophy: follow-up of two open-label phase 1/2 studies. Lancet (2015) 385(9967): 509-16. doi:10.1016/S0140-6736(14)61376-3

14. Haase A, Olmer R, Schwanke K, Wunderlich S, Merkert S, Hess C, et al. Generation of induced pluripotent stem cells from human cord blood. Cell Stem Cell (2009) 5(4):434-41. doi:10.1016/j.stem.2009.08.021 targeted genome engineering of the cells HLA-system. However, such approaches are not mature yet, and it remains to be demonstrated whether allotransplantation of such cells will result in long-term engraftment with very limited pharmacological immunosuppression.

\section{AUTHOR CONTRIBUTIONS}

The author confirms being the sole contributor of this work and approved it for publication.

\section{ACKNOWLEDGMENTS}

The author would like to thank Randi Diestel for the screening of international registries and both her and Anais Sahabian for proof reading the manuscript.

15. Schlaeger TM, Daheron L, Brickler TR, Entwisle S, Chan K, Cianci A, et al. A comparison of non-integrating reprogramming methods. Nat Biotechnol (2015) 33(1):58-63. doi:10.1038/nbt.3070

16. Olmer R, Lange A, Selzer S, Kasper C, Haverich A, Martin U, et al. Suspension culture of human pluripotent stem cells in controlled, stirred bioreactors. Tissue Eng Part C Methods (2012) 18(10):772-84. doi:10.1089/ten.TEC.2011.0717

17. Kropp C, Kempf H, Halloin C, Robles-Diaz D, Franke A, Scheper T, et al. Impact of feeding strategies on the scalable expansion of human pluripotent stem cells in single-use stirred tank bioreactors. Stem Cells Transl Med (2016) 5(10):1289-301. doi:10.5966/sctm.2015-0253

18. Burridge PW, Thompson S, Millrod MA, Weinberg S, Yuan X, Peters A, et al. A universal system for highly efficient cardiac differentiation of human induced pluripotent stem cells that eliminates interline variability. PLoS One (2011) 6(4):e18293. doi:10.1371/journal.pone.0018293

19. Saxena P, Heng BC, Bai P, Folcher M, Zulewski H, Fussenegger M. A programmable synthetic lineage-control network that differentiates human IPSCs into glucose-sensitive insulin-secreting beta-like cells. Nat Commun (2016) 7:11247. doi:10.1038/ncomms11247

20. Tao Y, Zhang SC. Neural subtype specification from human pluripotent stem cells. Cell Stem Cell (2016) 19(5):573-86. doi:10.1016/j.stem.2016.10.015

21. Lian X, Zhang J, Azarin SM, Zhu K, Hazeltine LB, Bao X, et al. Directed cardiomyocyte differentiation from human pluripotent stem cells by modulating Wnt/beta-catenin signaling under fully defined conditions. Nat Protoc (2013) 8(1):162-75. doi:10.1038/nprot.2012.150

22. Huang SX, Green MD, de Carvalho AT, Mumau M, Chen YW, D'Souza SL, et al. The in vitro generation of lung and airway progenitor cells from human pluripotent stem cells. Nat Protoc (2015) 10(3):413-25. doi:10.1038/ nprot.2015.023

23. Kempf H, Olmer R, Haase A, Franke A, Bolesani E, Schwanke K, et al. Bulk cell density and Wnt/TGFbeta signalling regulate mesendodermal patterning of human pluripotent stem cells. Nat Commun (2016) 7:13602. doi:10.1038/ ncomms 13602

24. Qi Y, Zhang XJ, Renier N, Wu Z, Atkin T, Sun Z, et al. Combined smallmolecule inhibition accelerates the derivation of functional cortical neurons from human pluripotent stem cells. Nat Biotechnol (2017) 35(2):154-63. doi:10.1038/nbt.3777

25. Gaj T, Gersbach CA, Barbas CF III. ZFN, TALEN, and CRISPR/Cas-based methods for genome engineering. Trends Biotechnol (2013) 31(7):397-405. doi:10.1016/j.tibtech.2013.04.004

26. Merkert S, Wunderlich S, Bednarski C, Beier J, Haase A, Dreyer AK, et al. Efficient designer nuclease-based homologous recombination enables direct PCR screening for footprintless targeted human pluripotent stem cells. Stem Cell Reports (2014) 2(1):107-18. doi:10.1016/j.stemcr.2013.12.003

27. Templin C, Zweigerdt R, Schwanke K, Olmer R, Ghadri JR, Emmert MY, et al. Transplantation and tracking of human-induced pluripotent stem cells in a pig model of myocardial infarction: assessment of cell survival, 
engraftment, and distribution by hybrid single photon emission computed tomography/computed tomography of sodium iodide symporter transgene expression. Circulation (2012) 126(4):430-9. doi:10.1161/ CIRCULATIONAHA.111.087684

28. Klug MG, Soonpaa MH, Koh GY, Field LJ. Genetically selected cardiomyocytes from differentiating embronic stem cells form stable intracardiac grafts. J Clin Invest (1996) 98(1):216-24. doi:10.1172/JCI118769

29. Di Stasi A, Tey SK, Dotti G, Fujita Y, Kennedy-Nasser A, Martinez C, et al. Inducible apoptosis as a safety switch for adoptive cell therapy. $N$ Engl J Med (2011) 365(18):1673-83. doi:10.1056/NEJMoa1106152

30. Valina C, Pinkernell K, Song YH, Bai X, Sadat S, Campeau RJ, et al. Intracoronary administration of autologous adipose tissue-derived stem cells improves left ventricular function, perfusion, and remodelling after acute myocardial infarction. Eur Heart J (2007) 28(21):2667-77. doi:10.1093/ eurheartj/ehm 426

31. Shiba Y, Fernandes S, Zhu WZ, Filice D, Muskheli V, Kim J, et al. Human ES-cell-derived cardiomyocytes electrically couple and suppress arrhythmias in injured hearts. Nature (2012) 489(7415):322-5. doi:10.1038/ nature11317

32. Chong JJ, Yang X, Don CW, Minami E, Liu YW, Weyers JJ, et al. Human embryonic-stem-cell-derived cardiomyocytes regenerate non-human primate hearts. Nature (2014) 510(7504):273-7. doi:10.1038/nature13233

33. Martens A, Rojas SV, Baraki H, Rathert C, Schecker N, Zweigerdt R, et al. Substantial early loss of induced pluripotent stem cells following transplantation in myocardial infarction. Artif Organs (2014) 38(11):978-84. doi:10.1111/aor.12268

34. Ronen D, Benvenisty N. Genomic stability in reprogramming. Curr Opin Genet Dev (2012) 22(5):444-9. doi:10.1016/j.gde.2012.09.003

35. Abyzov A, Mariani J, Palejev D, Zhang Y, Haney MS, Tomasini L, et al. Somatic copy number mosaicism in human skin revealed by induced pluripotent stem cells. Nature (2012) 492(7429):438-42. doi:10.1038/ nature11629

36. Tomasetti C, Li L, Vogelstein B. Stem cell divisions, somatic mutations, cancer etiology, and cancer prevention. Science (2017) 355(6331):1330-4. doi:10.1126/science.aaf 9011

37. Reardon S, Cyranoski D. Japan stem-cell trial stirs envy. Nature (2014) 513(7518):287-8. doi:10.1038/513287a

38. Mandai M, Watanabe A, Kurimoto Y, Hirami Y, Morinaga C, Daimon T, et al. Autologous induced stem-cell-derived retinal cells for macular degeneration. N Engl J Med (2017) 376(11):1038-46. doi:10.1056/NEJMoa 1608368

39. Baker M. Stem-cell pioneer bows out. Nature (2011) 479(7374):459. doi:10.1038/479459a
40. Blin G, Nury D, Stefanovic S, Neri T, Guillevic O, Brinon B, et al. A purified population of multipotent cardiovascular progenitors derived from primate pluripotent stem cells engrafts in postmyocardial infarcted nonhuman primates. J Clin Invest (2010) 120(4):1125-39. doi:10.1172/JCI40120

41. Kempf H, Olmer R, Kropp C, Ruckert M, Jara-Avaca M, Robles-Diaz D, et al. Controlling expansion and cardiomyogenic differentiation of human pluripotent stem cells in scalable suspension culture. Stem Cell Reports (2014) 3(6):1132-46. doi:10.1016/j.stemcr.2014.09.017

42. Hattori F, Chen H, Yamashita H, Tohyama S, Satoh YS, Yuasa S, et al. Nongenetic method for purifying stem cell-derived cardiomyocytes. Nat Methods (2010) 7(1):61-6. doi:10.1038/nmeth.1403

43. Dubois NC, Craft AM, Sharma P, Elliott DA, Stanley EG, Elefanty AG, et al. SIRPA is a specific cell-surface marker for isolating cardiomyocytes derived from human pluripotent stem cells. Nat Biotechnol (2011) 29(11):1011-8. doi:10.1038/nbt.2005

44. Tohyama S, Hattori F, Sano M, Hishiki T, Nagahata Y, Matsuura T, et al. Distinct metabolic flow enables large-scale purification of mouse and human pluripotent stem cell-derived cardiomyocytes. Cell Stem Cell (2013) 12(1):127-37. doi:10.1016/j.stem.2012.09.013

45. Jung JJ, Husse B, Rimmbach C, Krebs S, Stieber J, Steinhoff G, et al. Programming and isolation of highly pure physiologically and pharmacologically functional sinus-nodal bodies from pluripotent stem cells. Stem Cell Reports (2014) 2(5):592-605. doi:10.1016/j.stemcr.2014.03.006

46. Lee JH, Protze SI, Laksman Z, Backx PH, Keller GM. Human pluripotent stem cell-derived atrial and ventricular cardiomyocytes develop from distinct mesoderm populations. Cell Stem Cell (2017) 21(2):179-94.e4. doi:10.1016/ j.stem.2017.07.003

47. Gornalusse GG, Hirata RK, Funk SE, Riolobos L, Lopes VS, Manske G, et al. HLA-E-expressing pluripotent stem cells escape allogeneic responses and lysis by NK cells. Nat Biotechnol (2017) 35(8):765-72. doi:10.1038/nbt.3860

Conflict of Interest Statement: The author declares that the research was conducted in the absence of any commercial or financial relationships that could be construed as a potential conflict of interest.

The reviewer PH and handling Editor declared their shared affiliation.

Copyright $\odot 2017$ Martin. This is an open-access article distributed under the terms of the Creative Commons Attribution License (CC BY). The use, distribution or reproduction in other forums is permitted, provided the original author(s) or licensor are credited and that the original publication in this journal is cited, in accordance with accepted academic practice. No use, distribution or reproduction is permitted which does not comply with these terms. 